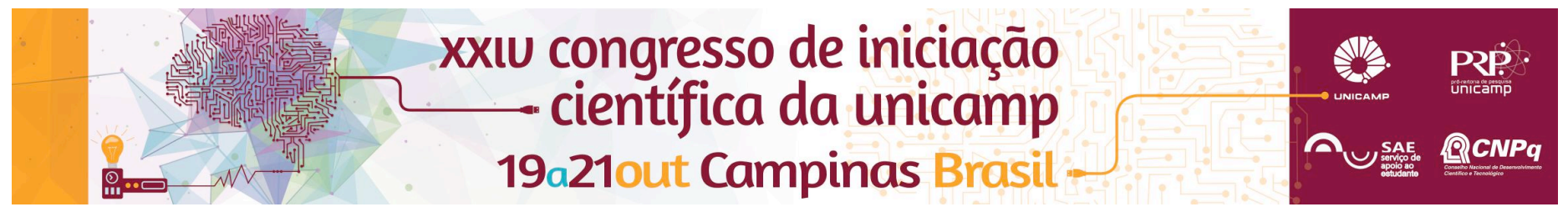

\title{
Evaluation of the pit and fissure sealant wettability
}

\author{
Giovana C. Magrini*, Matheus da S. Cancilieri, Taiza M. dos Santos, Ana Elisa D. de Faria, Kelly M. S. Moreira, \\ Regina M. Puppin-Rontani.
}

\begin{abstract}
Since the effectiveness of occlusal sealing is related to proper seal of the surface, a higher wettability of the sealant would assist in their scattering and avoid the bubble formation, allowing greater adhesion to the enamel structure. So, this study aimed to evaluate the wettability of different resinous sealants front of the smooth and rough glass. The sealants used were: Fluorshield, Helioseal Clear and Defense Chroma Angie. The material wettability was measured by contact angle (sessile drop), using Digidrop goniometer and considering the following groups: 1 . Smooth surface; 2. Rough surface of a glass slide. Data were submitted to factorial ANOVA (material $x$ surface) and Tukey's test $(p<0.05$ ), using Assistat 7.7 software. There was significant interaction between the materials and surfaces studied $(p<0.01)$. The Helioseal Clear sealant showed the highest wettability on rough glass. Fluorshield and Defense Chroma Angie sealants when applied on smooth glass surface showed lower wettability. Therefore, different sealants exhibit different wettability levels and the type of surface affect this property. The higher wettability was found in the Helioseal Clear sealant when applied onto the rough surface of a glass slide.
\end{abstract}

\section{Key words:}

Dental Carie, Pit and Fissure Sealants, Wettability.

\section{Introduction}

Pit and fissure sealants have shown efficacy in the further prevention of carious lesions on oclusal surfaces. ${ }^{1}$ Since the effectiveness of occlusal sealing seems to be related to proper seal of the surface, ${ }^{2}$ the greater wettability of the sealant would assist in their scattering and avoid the formation of bubbles, allowing greater adhesion to the enamel structure. So, this study aimed to evaluate the effect of different sealants and different smoothable surfaces of glass on the wettability physical property.

\section{Results and Discussion}

The wettability of the materials (Table 1) was measured by contact angle (Figure 1) front of the smooth and rough surface of a glass slide (<angle $=>$ wettability).

Table 1. Sealants used in the study.

\begin{tabular}{cccc}
\hline Material & Manufacturers & Load & Fluoride \\
\hline Fluorshield (FL) & Dentsply & With & Sodium \\
(EUA, DE) & load & fluoride \\
Defense Chroma & Angelus (Brasil, & With & Sodium \\
Angie (DC) & PR) & load & fluoride \\
Helioseal Clear & Ivoclar Vivadent & Without & Without \\
(HC) & (EUA, FL) & load & fluoride \\
\hline
\end{tabular}

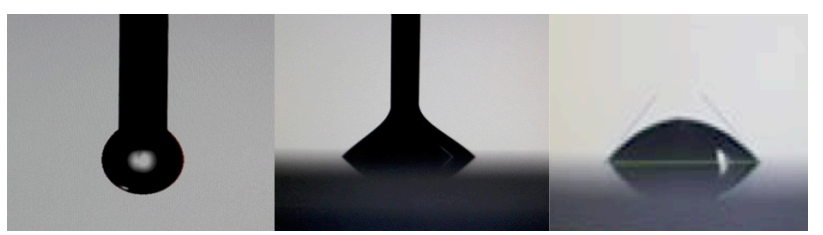

Figure 2. A) Drop formation. B) Drop dispensed. C) Contact angle (Digidrop goniometer). ( $n=10)$

There was interaction between materials and surfaces studied $(p<0.01)$.

The HC applied over the rough surface showed the highest wettability. Smaller wettability was observed for FL and DC applied over the smooth surface (Gráfico 1).

These results reveal new prospects to researchs about the efficacy of pit and fissure sealants, since the adhesion of these materials to the surface can be related to difference in the wettability physical property.

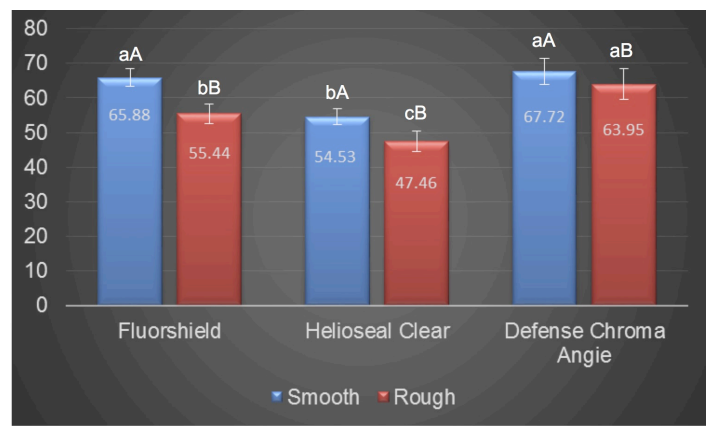

${ }^{*}$ Different capital letters mean significant difference between surface type; Different small letters mean significant difference between sealants.

Graph 1. Sealant contact angle on glass surfaces: mean and standard deviation.

\section{Conclusions}

The sealants showed different wettability, which was influenced by type of surface and sealant composition.

The higher wettability was found for HC applied over rough surface of a glass slide.

\section{Acknowledgement}

This research was supported by CNPq - National Council for Scientific and Technological Development.

\footnotetext{
Ahovuo-Saloranta A, Hiiri A, Nordblad A, Mäkelä M, Worthington H. Pit and fissure sealants for preventing dental decay in the permanent teeth of children and adolescents. Cochrane Database Syst Rev. 2013 Mar 28;3:CD001830. doi: $10.1002 / 14651858$.

${ }^{2}$ Reddy VR, Chowdhary N, Mukunda KS, Kiran NK, Kavyarani BS, Pradeep MC. Retention of resin-based filled and unfilled pit and fissure sealants: A comparative clinical study. Contemp Clin Dent. 2015 Mar;6(S1):18-23.
} 